\title{
Human Anti-Ricin Antibody
}

National Cancer Institute

\section{Source}

National Cancer Institute. Human Anti-Ricin Antibody. NCI Thesaurus. Code C107430.

A human antibody which recognizes ricin epitopes. These antibodies often develop in

response to treatment with immunotoxins that are comprised of ricin

immunoconjug ates and limit the effectiveness of the treatment. 